REMIPE

\title{
Avaliação do nível de maturidade para a geração de empreendimentos inovadores com metodologia CERNE: estudo das incubadoras do Extremo Sul/RS
}

\section{Maturity level assessment for the generation of innovative enterprises with CERNE methodology: study of the incubators in the Extreme South/RS}

\author{
Recebido: 15/05/2019 - Aprovado:01/06/2020 - Publicado:01/07/2020 \\ Processo de Avaliação: Double Blind Review
}

\author{
Priscila Nesello \\ pri.nesello@gmail.com \\ Universidade Federal de Pelotas, Brasil \\ https://orcid.org/0000-0002-9312-463X \\ Cristiane Vasconcelos da Rosa \\ cristianevasconcelosrosa@gmail.com \\ Universidade Federal de Pelotas, Brasil \\ https://orcid.org/0000-0003-1772-6388
}

\section{RESUMO}

Este artigo tem como objetivo avaliar o nível de maturidade das incubadoras do Extremo Sul do Rio Grande do Sul, com a utilização da Metodologia do Centro de Referência para Apoio a Novos Empreendimentos (CERNE). O CERNE é um modelo direcionado para resultados e promove a melhoria na qualidade dos processos das incubadoras, aumentando o potencial de geração de empreendimentos inovadores. O modelo possui oito processos-chave e trinta e três práticas-chave. A avaliação foi feita em duas incubadoras de base tecnológica da cidade de Pelotas-RS, a CONECTAR vinculada à Universidade Federal de Pelotas (UFPEL) e a CIEMSUL vinculada à Universidade Católica de Pelotas (UCPEL). A pesquisa foi desenvolvida em duas etapas, qualitativa e quantitativa. A etapa qualitativa foi realizada por meio de entrevistas presenciais. A etapa quantitativa foi realizada com a aplicação de questionário, baseado nos processos-chave do CERNE NÍVEL 1. Ambas as etapas foram realizadas com a participação dos coordenadores das incubadoras. Como resultado, foi possível identificar que as incubadoras estudadas encontram-se na fase de implantação do CERNE NÍVEL 1. A contribuição deste estudo está em evidenciar a importância da articulação de um planejamento de implantação das práticas do modelo CERNE, com vistas a aperfeiçoar o atendimento aos incubados por meio de procedimentos que possibilitem uma melhor gestão 
administrativa, alinhando ações para complementar e disponibilizar uma base referencial de produtos, serviços e processos.

Palavras-chave: incubadora, CERNE, práticas-chave.

\section{ABSTRACT}

This article aims at assessing the level of maturity of incubators in the extreme south of Rio Grande do Sul, using the methodology of the Reference Center to Support New Ventures (CERNE). CERNE is a results-oriented model and promotes the improvement in the quality of incubator processes, increasing the potential for generating innovative ventures. The model has eight key processes and thirty-three key practices. The evaluation was carried out in two technology-based incubators in the city of Pelotas-RS, CONECTAR linked to the Federal University of Pelotas (UFPEL) and CIEMSUL linked to the Catholic University of Pelotas (UCPEL). The research was developed in two stages, qualitative and quantitative. The qualitative step was performed through face-to-face interviews. The quantitative stage was carried out with the application of a questionnaire, based on the key processes of CERNE LEVEL 1. Both steps were carried out with the participation of the coordinators of the incubators. As a result, it was possible to identify that the incubators studied are in the phase of implantation of CERNE LEVEL 1. The contribution of this study is to highlight the importance of the articulation of an implantation planning of the practices related to the CERNE model, with a view to improve the service incubated through procedures that enable better administrative management, aligning actions to complement and provide a reference base of products, services and processes.

Key-words: incubator, CERNE, key practices.

\section{INTRODUÇÃO}

Atualmente, o empreendedorismo e a cultura de inovação têm ocupado um papel de extrema importância para a economia do país. A carreira empreendedora vem sendo estimulada nas universidades para que o público tenha uma visão ampla do quanto pode contribuir para a sociedade, tendo como base as atividades oferecidas por essas instituições. Ao ingressar no mercado, o empreendedor busca uma oportunidade de colocar em prática sua vivência em sala 
de aula, através da abertura de um negócio próprio e então surgem inúmeras dificuldades de gestão na prática.

Pesquisa feita pelo Serviço Brasileiro de Apoio às Micro e Pequenas Empresas (SEBRAE2016) relata os principais fatores que contribuem para a sobrevivência ou mortalidade das empresas no Brasil. Esses fatores, nos 2 primeiros anos, são: situação antes da abertura, planejamento do negócio, gestão do negócio e a capacitação dos donos em gestão empresarial.

Tendo em vista esses fatores, surge a necessidade de um acompanhamento aos novos empreendedores durante esses primeiros 2 anos, onde entra o suporte das incubadoras vinculadas às próprias universidades, na maioria das vezes, situadas dentro de parques tecnológicos. O crescimento dessas incubadoras no Brasil seguiu uma curva exponencial, passando de duas unidades no ano de 1988 para 339 unidades no ano de 2005, segundo dados da ANPROTEC (2005). Estudos mais recentes apontam que o número de incubadoras em 2012 firmou em 384. Dessas incubadoras, $40 \%$ atuam com empresas de base tecnológica (ANPROTEC; MCTI, 2012).

Esse valor expressivo das incubadoras de empresas de base tecnológica, frente às demais incubadoras reflete a importância que essas organizações apresentam por lidarem com o empreendedorismo tecnológico e o desenvolvimento tecnológico. Essas são atividades alvo de políticas macroeconômicas no Brasil, intensificadas na última década com a sanção, por parte do governo brasileiro, da Lei da Inovação, em 2004. Esse incentivo ao desenvolvimento tecnológico e ao crescimento apresentado pelas incubadoras leva a considerar, também, a qualidade do crescimento, que pode ser replicado na maturidade das incubadoras de empresas e no desenvolvimento de novos empreendimentos inovadores.

Aplicar o modelo de avaliação ao nível de maturidade contribui para identificar o caminho que as incubadoras estão seguindo e quais processos devem ser modificados ou sistematizados. Sendo assim, proporciona a eficácia dos resultados ao prever o desperdício de recursos financeiros e tempo investido, contribuindo para o crescimento de 2.640 empresas incubadas, 16.394 postos de trabalhos nessas empresas e $\mathrm{R} \$ 533$ milhões em faturamento nas empresas incubadas (ANPROTEC; MCTI, 2012). Dessa forma, percebe-se a importância de se estudar a aplicação do modelo de avaliação de maturidade nessas organizações, o que justifica o desenvolvimento desta pesquisa.

$\mathrm{O}$ ambiente de pesquisa caracterizou-se com o estudo em duas incubadoras de base tecnológica, a CONECTAR vinculada à UFPel e a CIEMSUL vinculada à UCPel. O objetivo

REMIPE- Revista de Micro e Pequenas Empresas e Empreendedorismo da Fatec Osasco V. $6 \mathrm{~N}^{\circ} 2$ jul.-dez. 2020. 
foi avaliar o nível de maturidade das incubadoras do Extremo Sul do Rio Grande do Sul. Por fim, a pergunta principal desta pesquisa é: qual o nível de maturidade das incubadoras de base tecnológica no Extremo Sul?

O artigo está estruturado em seis seções sendo esta a primeira. A segunda seção aborda o marco teórico da pesquisa, considerando os aspectos conceituais que envolvem o tema inovação e empreendedorismo, ecossistema empreendedor e o modelo teórico utilizado na pesquisa. A terceira seção apresenta a caracterização do ambiente da pesquisa, enquanto a seção quatro apresenta os aspectos metodológicos da pesquisa, os resultados e a discussão dos resultados. Por fim, a seção cinco apresenta as considerações finais da pesquisa e a seção seis reúne as referências bibliográficas utilizadas como suporte teórico.

\section{INOVAÇÃO E EMPREENDEDORISMO}

De acordo com Plonski (2006, p.25), inovação é a "nova ideia implementada com sucesso produzindo efeitos econômicos ou sociais". Segundo Moraes (2007, p.4), a inovação tecnológica representa "um processo de aprendizagem contínuo e cumulativo das empresas para melhorar produtos/serviços, processos e formas de gestão, modelo de interação social, de modo a aumentar a produtividade, conhecimento e competitividade". É preciso considerar que a inovação não ocorre num vazio e não se limita a uma empresa individual. Isso resulta em implicações políticas, econômicas e sociais, seus efeitos se estendem por toda a rede na qual esteja inserida. Segundo Drucker (1985), inovação é uma ferramenta própria dos empreendedores e é por ela que eles pesquisam as mudanças, enxergando as oportunidades para um negócio ou serviço diferente.

Conforme Bessant e Tidd (2015), o problema de um indivíduo é a oportunidade de outro, e assim a natureza da inovação é fundamental. O empreendedorismo, por sua vez, ocasiona uma mistura de visão, paixão, energia, entusiasmo, insight, julgamento e muito trabalho, o que permite que boas ideias se tornem realidade. Segundo Dornelas (2008), o primeiro exemplo de empreendedorismo pode ser visto quando Marco Polo tentou estabelecer uma rota comercial para o Oriente. Marco Polo assinou um contrato com um homem que tinha dinheiro, hoje conhecido como capitalista, para então vender as mercadorias desse, assumindo assim todos os riscos calculados que hoje conhecemos como parte de um perfil empreendedor.

REMIPE- Revista de Micro e Pequenas Empresas e Empreendedorismo da Fatec Osasco 
Empreendedorismo é o envolvimento de pessoas e processos que, em conjunto, levam a transformação de ideias em oportunidades, enfatizando assim de forma mais geral o real objetivo do empreendedorismo, que é gerar oportunidades (DORNELAS 2008). Para Dornelas (2008), o empreendedor é aquele que faz as coisas acontecerem, antecipa os fatos e tem uma visão futura da organização. Já para Chiavenato (2007, p. 36), “o empreendedor é a pessoa que inicia ou opera um negócio para realizar uma ideia ou projeto pessoal assumindo riscos e responsabilidades e inovando continuamente". Os termos inovação e empreendedorismo têm ganhado ênfase no contexto atual, pelo fato de que em nossa economia, produção e serviços devem se adaptar às mudanças para suprir as necessidades da sociedade.

\subsection{Ecossistema Empreendedor: Parques Tecnológicos, Incubadoras, Startups e Aceleradoras.}

Um ecossistema empreendedor é formado por empresas, governo, instituições de pesquisa e ensino, incubadoras, aceleradoras, associações de classe, prestadores de serviço e empreendedores. Ele surge a partir de cidades inteligentes que buscam soluções para seus problemas e geram novas oportunidades para a sociedade. Essa por sua vez, tem pessoas com os mais diversificados talentos e capacidades, e os utilizam como ferramenta para melhorar a qualidade de vida dos locais onde estão inseridos (AUDY, 2016).

Um fator preponderante para captação e suporte ao incentivo do desenvolvimento desses talentos é a criação de parques tecnológicos. A partir da congregação de instituições públicas e privadas, os parques, com foco em empresas inovadoras, startups de base tecnológica, centros tecnológicos, institutos de pesquisas e universidades constituem importantes instrumentos para o desenvolvimento de ambientes inovadores (BELLAVISTA; SANZ, 2009). São diversas as denominações existentes para esses parques tecnológicos: polos de tecnologia, centros de alta tecnologia, centros de incubação, tecnoparques ou cidades científicas, parques científicos e tecnológicos. Esses buscam fomentar a transferência tecnológica e a inovação, aumentando a competitividade de empresas, regiões e até mesmo nações (HASSINK; HU, 2012).

No Brasil, os primeiros incentivos para fomentar o desenvolvimento de centros de inovação tiveram início na década de 1980, com a criação do Programa Brasileiro de Parques Tecnológicos pelo Conselho Nacional de Desenvolvimento Científico e Tecnológico (CNPq). Esse programa visava modificar a realidade econômica do país, por meio de um direcionamento de recursos e esforços no aprimoramento e na geração de tecnologias (PLONSKI, 2010). Dados os desafios impostos pela falta de uma cultura direcionada à inovação e pelo baixo número de 
iniciativas inovadoras no território nacional, os projetos de parques tecnológicos da época não tiveram o impacto dos grandes centros mundiais de inovação. Seus resultados foram modestos, embora de relevância estratégica, ao originar as primeiras incubadoras de empresas brasileiras (ABDI; ANPROTEC, 2008).

Com o passar do tempo, novos incentivos foram lançados para a implantação de parques científicos e tecnológicos no Brasil. Os objetivos desses parques tornaram-se mais abrangentes, com desenvolvimento não somente tecnológico, como também econômico e social (ABDI; ANPROTEC, 2008). Assim, com a criação de marcos legais de apoio ao processo de inovação, a exemplo da Lei da Inovação, em 2004, um novo impulso foi dado ao desenvolvimento dos parques científicos e tecnológicos nacionais. Outro importante avanço ocorreu com a fundação do Programa Nacional de Incubadoras e Parques Tecnológicos (PNI) pelo Ministério da Ciência, Tecnologia e Inovação (MCTI) (ANPROTEC, 2014).

O PNI tem por objetivos fomentar a consolidação e o surgimento de parques tecnológicos e incubadoras de empresas, que contribuam para estimular e acelerar o processo de criação de micro e pequenas empresas. Essas são caracterizadas pelo elevado conteúdo tecnológico de seus produtos, processos e serviços, bem como por intensa atividade de inovação tecnológica e pela utilização de modernos métodos de gestão (MCTI, 2009).

Na década 1980, o movimento das incubadoras de empresas começou no Brasil. Foram apoiadores, o Conselho Nacional de Desenvolvimento Científico e Tecnológico (CNPq), agências como a Financiadora de Estudos e Projetos (FINEP) e a Organização dos Estados Americanos (OEA) no plano supranacional. Estudos apoiados por essas agências levaram à constituição, em 1987, da Associação Nacional de Entidades Promotoras de Empreendimentos de Tecnologia Avançada (ANPROTEC), cujo objetivo tem sido a articulação com organismos governamentais e não-governamentais, visando ao desenvolvimento de Incubadoras e Parques Tecnológicos no País. O surgimento desse arranjo esteve ligado à existência de um vácuo institucional, identificando assim uma lacuna onde fosse promovida a relação entre o ambiente acadêmico e o setor empresarial (OLIVEIRA, 2003).

Para ANPROTEC, entidade que coordena o setor no país, as incubadoras:

Designam empreendimentos que ofereçam espaço físico, por tempo limitado, para a instalação de empresas de base tecnológica e/ou tradicional, e que disponham de uma equipe técnica para fornecer suporte e consultoria a estas empresas (ANPROTEC, 2001, p. 28). 
Com a finalidade de apoiar e financiar o sistema brasileiro de ciência, tecnologia e inovação, a Finep, organização pública ligada ao Ministério da Ciência, Tecnologia e Inovação (MCTI), tem oferecido recursos reembolsáveis e não-reembolsáveis. Esses recursos abrangem todas as etapas e dimensões do ciclo de desenvolvimento científico e tecnológico: pesquisa básica, pesquisa aplicada, melhoria e desenvolvimento de produtos, serviços e processos.

Com base na National Business Incubation Association - NBIA (2012), a incubação de empresas envolve um processo de apoio a negócios. Esse processo acelera o desenvolvimento de empresas startups com a oferta de recursos e serviços diversificados pelas equipes das incubadoras ou de sua rede de parceiros. Segundo Blank (2014), startups são definidas como organizações temporárias em busca de um modelo de negócio repetível e escalável. Para Ries (2012), startups tratam-se de um tipo de empresa como uma instituição humana projetada para criar novos produtos e serviços sob condições de extrema incerteza. De acordo com essas definições, identifica-se que uma startup necessita de aporte tanto de capital financeiro como intelectual para esse período inicial, onde essas incertezas irão surgir a qualquer momento.

Além das incubadoras, as aceleradoras entram nesse cenário para oferecer suporte adequado. A diferença entre as incubadoras e as aceleradoras está no fato de que as primeiras dão suporte às empresas nascentes, startups na fase inicial do ciclo de negócios, por períodos mais longos 2 ou 3 anos. Já as aceleradoras preparam as startups para o crescimento durante um período menor de tempo até 6 meses, fazendo investimento de risco para adquirir uma parte (5 a 15\%) das ações da empresa acelerada (LIMEIRA, 2014).

\subsection{Modelo de Maturidade CERNE}

Em 2009, foi criado pelo Serviço Brasileiro de Apoio às Micro e Pequenas Empresas (SEBRAE) e pela ANPROTEC, o Centro de Referência para Apoio a Novos Empreendimentos (CERNE). O CERNE é uma plataforma que visa promover a melhoria expressiva nos resultados das diferentes incubadoras de diferentes setores de atuação abrangendo cinco dimensões: empreendedor, tecnologia, capital, mercado e gestão. Para isso, determina boas práticas a serem adotadas em diversos processos-chave, que estão associados a níveis de maturidade. Cada nível de maturidade contribui para que a incubadora possa constantemente evoluir sua capacidade de apoiar novos empreendimentos. Os processos chave que compõem os diferentes níveis de maturidade, quando relacionados ao apoio aos empreendimentos, levam sempre em consideração cinco elementos fundamentais ao desenvolvimento dos negócios: pessoal (empreendedor enquanto pessoa), gestão, mercado, finanças e tecnologia (ANPROTEC, 2011).

REMIPE- Revista de Micro e Pequenas Empresas e Empreendedorismo da Fatec Osasco V. $6 \mathrm{~N}^{\circ} 2$ jul.-dez. 2020. 
O CERNE é uma iniciativa pioneira que propõe um modelo de excelência em gestão. O modelo é voltado para resultados e visa diminuir a variabilidade entre as incubadoras, aumentar a qualidade dos processos e gerar mais e melhores empreendimentos inovadores (SEBRAE, 2015). De acordo com a ANPROTEC (2014), o objetivo do modelo CERNE é de suprir as necessidades das incubadoras e proporcionar a capacidade de desenvolver empreendimentos de sucesso, uma vez que a incubadora passa a atuar de maneira proativa na geração do desenvolvimento sustentável, com base na inovação. Ao estipular procedimentos, o modelo CERNE busca proporcionar uma rede de soluções que capacitem e desenvolvam empresas e empreendedores, de modo a gerar negócios bem-sucedidos. O CERNE é norteado por um conjunto de princípios sobre os quais os processos-chaves e as práticas estão fundamentadas. Os princípios do modelo CERNE estão detalhados a seguir:

- Foco nos empreendimentos: esse princípio salienta que o foco da incubadora deve ser agregar valor aos empreendimentos apoiados;

- Foco nos processos: de acordo com esse princípio, são os processos utilizados pela incubadora que influenciam nos resultados obtidos tanto da incubadora quanto das empresas incubadas e, portanto, merecem toda a atenção por parte da incubadora;

- Ética: as ações da incubadora e das empresas incubadas necessitam estar em sintonia com os valores da sociedade;

- Sustentabilidade: esse princípio destaca que a incubadora deve ser viável economicamente, justa socialmente e correta ambientalmente;

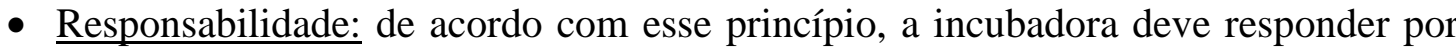
seus atos e omissões, agindo ativamente para melhorar a sociedade em que atua;

- Melhoria contínua: esse princípio estabelece que a incubadora deve melhorar continuamente seus processos e resultados;

- Desenvolvimento humano: esse princípio estipula que a incubadora deve dar prioridade à evolução pessoal e profissional salientando a autogestão e também o autocontrole;

- Gestão transparente e participativa: de acordo com esse princípio, as ações da incubadora devem ser realizadas de maneira colaborativa. E, além disso, todos os processos e resultados devem ser transparentes aos distintos atores do processo de inovação.

De acordo com ANPROTEC (2015), o modelo CERNE é dividido em quatro categorias: CERNE 1 - Empreendimento; CERNE 2 - Incubadora; CERNE 3 - Rede de Parceiros e, CERNE 4 Melhoria Contínua. O modelo Cerne dispõe de níveis de maturidade diretamente 
relacionados à evolução do desenvolvimento da incubadora, sendo que cada nível de maturidade representa uma etapa a ser alcançada pelas incubadoras.

No nível CERNE 1, todos os sistemas são diretamente relacionados ao desenvolvimento dos empreendimentos, havendo sistemas de qualificação, assessoria e seleção, bem como gestão financeira, gestão de infraestrutura física e tecnologia. Estando nesse nível, a incubadora revela que tem condições para prosperar e selecionar boas ideias, podendo transformá-las em empreendimentos inovadores de sucesso.

O foco do nível CERNE 2, por sua vez, é o de garantir uma gestão atuante da incubadora como uma organização, utilizando todos os sistemas para uma gestão focada em resultados. Além disso, para estar nesse nível a incubadora deve também manter o foco no que foi proposto no nível CERNE 1.

Já no nível CERNE 3, o objetivo é o de consolidar uma rede de parceiros, buscando ampliar a probabilidade de negócios apoiados bem-sucedidos, bem como reforçar o papel da incubadora como um dos "nós" da rede de participantes envolvidos no processo de inovação. Além disso, deve-se manter o foco também na estrutura criada no CERNE 1 e no CERNE 2.

O nível CERNE 4, consequentemente, parte da estrutura implantada nos níveis anteriores (CERNE 1, CERNE 2 e CERNE 3). No entanto, nesse nível a incubadora já possui maturidade para consolidar seu sistema de gestão, passando assim, a gerar inovações em seus próprios processos.

Além dos níveis de maturidade da incubadora, o Modelo CERNE também apresenta um conjunto de "processos-chaves" para cada um dos níveis de maturidade. Esses processos procuram garantir que a incubadora esteja utilizando todas as boas práticas relacionadas a determinado nível de maturidade. Para cada nível de maturidade, o modelo conta ainda com "práticas-chave". As práticas indicam o nível de evolução de cada processo implantado em um dado nível. A seguir são apresentadas as práticas constantes no modelo:

- Prática Inicial: estágio mais simples de uma prática refere-se à realização e ao registro das atividades relacionadas ao processo;

- Prática Definida: é o segundo estágio da evolução das práticas-chaves, sendo esse o estágio em que a incubadora planeja as atividades relacionadas à prática para os próximos 12 meses;

- Prática Estabelecida: É o terceiro estágio e se refere ao nível onde são criados indicadores para monitorar os resultados obtidos com a prática; 
REMIPE

- Prática Sistematizada: é o quarto e último nível das práticas-chaves, no qual é realizada a reunião crítica para avaliação da prática em questão, tendo como base os resultados dos indicadores, aprimorando a prática em questão.

A seguir, a Figura 1 apresenta o Modelo Cerne em termos de seus níveis, processos e práticas:

Figura 1 - Evolução da implantação CERNE

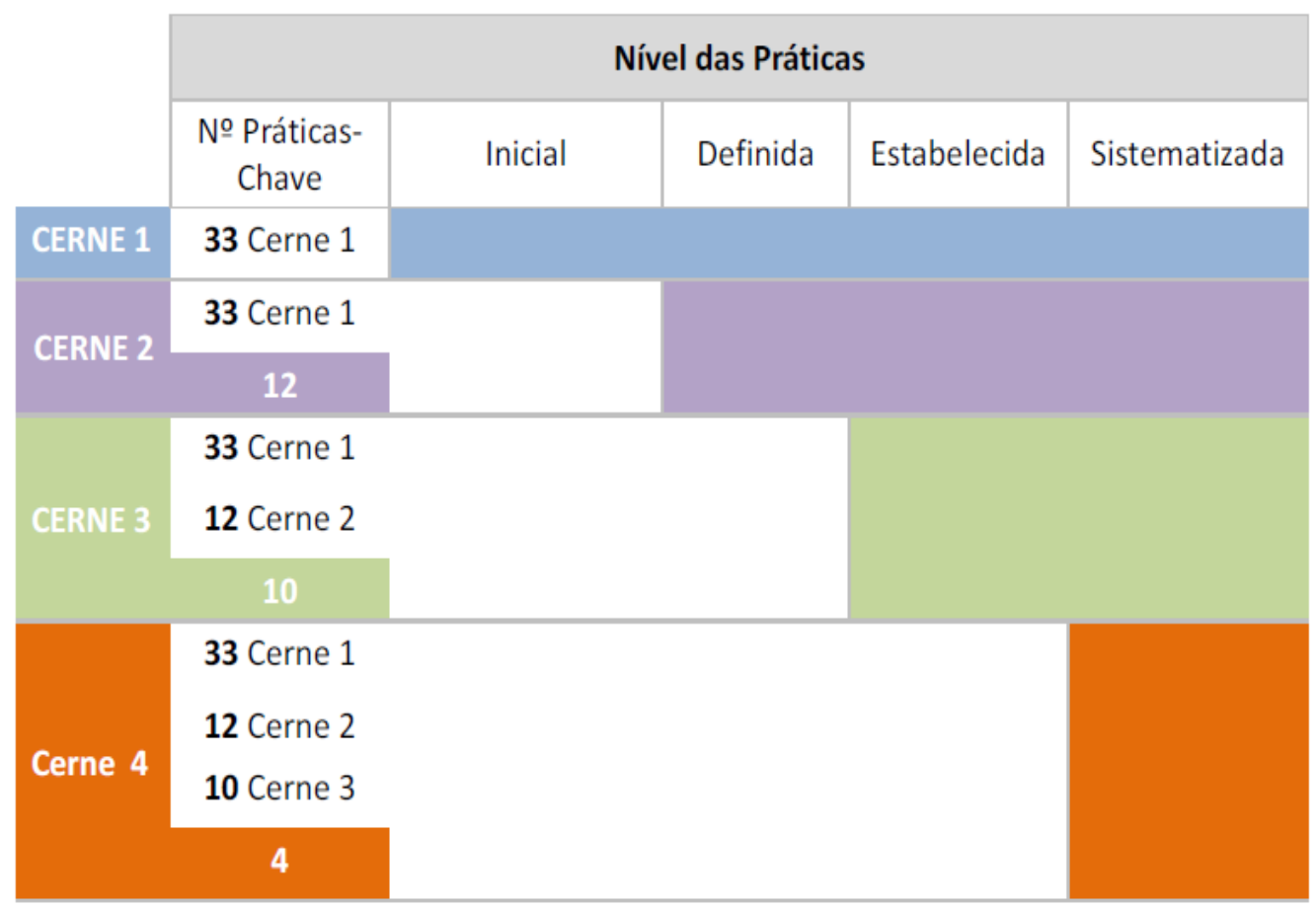

Fonte: CERNE (2013).

A lógica que fundamenta a relação entre os níveis de maturidade e a evolução das práticas-chaves incentiva as incubadoras a implantarem e desenvolverem a melhoria contínua de seus processos. Assim, ao detalhar os sistemas que compõem o modelo CERNE, tem-se que em cada nível de maturidade há processos-chave específicos e suas respectivas práticas-chave.

\section{CARACTERIZAÇÃO DO AMBIENTE DE PESQUISA}

A seguir serão apresentados dados das incubadoras tomadas como base para realização deste estudo, respectivamente, CONECTAR e CIEMSUL. 


\subsection{Incubadora CONECTAR}

Segundo o site da CONECTAR, é a primeira Incubadora de Base Tecnológica da Universidade Federal de Pelotas, cuja existência está regulamentada pela resolução $\mathrm{N}^{\circ} 11$ de 08 de agosto de 2015. A Conectar, via convênio com a Fundação de apoio Delfim Mendes Silveira, viabiliza a operacionalização dos processos de pré-incubação, incubação e pósincubação, desde a sua origem em janeiro de 2015.

A Incubadora de Base Tecnológica é um ambiente formado para abrigar empresas cujos processos, produtos ou serviços são gerados a partir de resultados de pesquisas básicas ou aplicadas nos quais a ciência e a tecnologia geram inovação e representam valor agregado. A CONECTAR está alocada no Parque Tecnológico de Pelotas e, atualmente, oferece suporte para 6 empresas incubadas.

\subsection{Incubadora CIEMSUL}

Segundo o site da CIEMSUL, a incubadora nasceu da vontade de ter na Universidade Católica de Pelotas um centro de empreendedorismo para oportunizar a geração de ideias inovadoras da comunidade. Dessa forma, em 30 de junho de 2000, foi criada a incubadora empresas multisetoriais pela UCPEL.

A incubadora tem como atribuições dar suporte a potenciais empreendedores, amparar novas empresas, fortalecer novos empreendimentos com uma infraestrutura de apoio e proporcionar a criação e consolidação de empreendimentos. A incubadora está alocada no Parque Tecnológico de Pelotas e possui 9 empresas incubadas.

O CIEMSUL está subordinado ao EDR - Escritório de Desenvolvimento Regional, órgão vinculado à Reitoria da Universidade Católica de Pelotas. A sua missão é "investigar a verdade, produzir e transmitir o conhecimento para formar seres humanos, profissionais éticos e competentes, orientados pelos valores cristãos, a serviço da pessoa e da sociedade".

\section{METODOLOGIA}

Este estudo se desenvolveu em duas etapas: (1) qualitativa e exploratória, com a realização de entrevistas com os coordenadores de duas incubadoras do Extremo Sul do Rio 
Grande do Sul; (2) quantitativa e descritiva, com o preenchimento de questionários onde os respectivos administradores das incubadoras avaliaram as práticas-chave de seus processos, a luz do modelo CERNE NÍVEL 1.

Em relação à etapa qualitativa e exploratória, foram realizadas entrevistas presenciais, com roteiro semiestruturado e gravação em áudio das falas dos dois coordenadores das incubadoras. Cada entrevista teve duração aproximada de 40 minutos. Os dados coletados foram analisados com base na técnica de análise de conteúdo e categorizados a priori de acordo com os processos-chave do método CERNE NÍVEL 1. Segundo Bardin (2011, p.47), a análise de conteúdo é "um conjunto de técnicas de análise das comunicações visando a obter, por procedimentos sistemáticos e objetivos de descrição do conteúdo das mensagens, indicadores (quantitativos ou não) que permitam a inferência de conhecimentos relativos às condições de produção/recepção (variáveis inferidas) dessas mensagens”.

Em relação à etapa quantitativa e descritiva, foi desenvolvido no aplicativo Google Forms, um questionário do modelo CERNE NÍVEL 1. No questionário, os entrevistados avaliaram o estágio de maturidade dos processos das suas respectivas incubadoras, relacionando-os com os processos-chave do modelo. O questionário foi composto por 33 questões de múltipla escolha, referentes ao CERNE NÍVEL 1 - Empreendimento. Os processos-chave avaliados foram: sensibilização e prospecção, seleção, planejamento, qualificação, assessoria e consultoria, monitoramento, graduação e relacionamento com graduados e gerenciamento básico.

Posteriormente, os dados da etapa exploratória e descritiva foram analisados e confrontados para atender ao objetivo do artigo que é avaliar o nível de maturidade das incubadoras do Extremo Sul do Rio Grande do Sul, com a utilização da metodologia CERNE. Para completar o quadro de incubadoras do Extremo Sul, foi também contatada a incubadora INNOVATIO, de Rio Grande, porém não foi possível agendar entrevista e realizar a coleta de dados dessa incubadora.

\subsection{Análise de Dados}

A seguir, serão apresentados os resultados obtidos a partir das etapas exploratória e descritiva do estudo. 


\subsection{Etapa Exploratória}

Ao dar início à análise dos dados coletados através das entrevistas, percebe-se que as incubadoras possuem um tempo significativo de atuação entre 3 e 18 anos. Ambas possuem processo de pré-incubação, através de edital. Sua estrutura é composta por empresas préincubadas, incubadas e pós-incubadas em processo de graduação/desligamento.

A primeira incubadora analisada é a Incubadora de Base Tecnológica da Universidade Federal de Pelotas-CONECTAR. Ela foi fundada em 2015 e, atualmente, está alocada dentro do Parque Tecnológico de Pelotas. Seu foco é voltado para negócios, com viés tecnológico vinculado às pesquisas desenvolvidas dentro da universidade. A segunda incubadora analisada é o Centro de Incubação de Empresas da Região Sul- CIEMSUL. Vinculada à Universidade Católica de Pelotas-UCPel, ela foi fundada em 2000. Atualmente, alocada no Parque Tecnológico de Pelotas, seu foco são negócios inovadores de produtos e/ou serviços, captados através de projetos desenvolvidos dentro da universidade. No Quadro 1, são apresentados alguns trechos das entrevistas realizadas em relação aos processos-chave:

Quadro 1 - Práticas-chave Implementadas

\begin{tabular}{|l|l|l|}
\hline \multicolumn{1}{|c|}{ Processo-chave } & \multicolumn{2}{|c|}{ Incubadora } \\
\hline $\begin{array}{l}\text { Sensibilização } \\
\text { Prospecção }\end{array}$ & $\begin{array}{l}|c| \\
\text { e Civulgação através do site, fanpage e } \\
\text { em eventos da UFPel. } \\
- \text { Disponibilidade para visitas da } \\
\text { comunidade acadêmica. }\end{array}$ & $\begin{array}{l}\text { - Divulgação através do site e em sala } \\
\text { de aula. } \\
\text { - Disponibilidade para visitas da } \\
\text { comunidade acadêmica. }\end{array}$ \\
\hline Seleção & $\begin{array}{l}\text { - Processo de seleção através de edital. } \\
\text { - Banca avaliadora das propostas de } \\
\text { projetos iniciantes. } \\
\text { - Manual do incubado, direitos e deveres. } \\
\text { - Contrato de incubação. }\end{array}$ & $\begin{array}{l}\text { - Processo de seleção através de } \\
\text { edital. } \\
\text { - Banca avaliadora das propostas de } \\
\text { projetos iniciantes. } \\
\text { - Contrato de incubação. }\end{array}$ \\
\hline Planejamento & $\begin{array}{l}\text { - Mentoria de professores e profissionais } \\
\text { parceiros. }\end{array}$ & $\begin{array}{l}\text { - Mentoria de professores e } \\
\text { profissionais parceiros. }\end{array}$ \\
\hline Qualificação & $\begin{array}{l}\text { - Cursos, palestras e eventos sem } \\
\text { periodicidade. }\end{array}$ & $\begin{array}{l}\text { - Cursos, palestras e eventos sem } \\
\text { periodicidade. }\end{array}$ \\
\hline Assessoria e Consultoria & $\begin{array}{l}\text { - Consultoria e assessoria de professores } \\
\text { e profissionais parceiros. }\end{array}$ & $\begin{array}{l}\text { Consultoria e assessoria de } \\
\text { professores e profissionais parceiros. }\end{array}$ \\
\hline Monitoramento & $\begin{array}{l}\text { Avaliação trimestral feita pelo comitê } \\
\text { gestor. }\end{array}$ & $\begin{array}{l}\text { - Percepção feita pelo coordenador, } \\
\text { instrumento não definido. }\end{array}$ \\
\hline $\begin{array}{l}\text { Graduação } \\
\text { Relacionamento }\end{array}$ & $\begin{array}{l}\text { - Divulgação no site de mudança de } \\
\text { status. }\end{array}$ & $\begin{array}{l}\text { - Divulgação no site de mudança de } \\
\text { status. }\end{array}$ \\
\hline Gerenciamento básico & $\begin{array}{l}\text { Coordenada pela equipe gestora. } \\
\text { - Espaço para incubação, sala de } \\
\text { reuniões, auditório, equipamentos } \\
\text { multimídia, serviço de limpeza e serviço } \\
\text { de segurança. }\end{array}$ & $\begin{array}{l}\text { - Coordenada pelo gestor. } \\
\text { - Espaço para incubação, sala de } \\
\text { reuniões, auditório, equipamentos } \\
\text { multimídia, serviço de limpeza e } \\
\text { serviço de segurança. }\end{array}$ \\
\hline
\end{tabular}

Fonte: Elaborado pelas autoras. 
As questões abordadas com os entrevistados, por meio do roteiro semiestruturado, abrangem desde o foco principal das incubadoras, quanto ao seu processo de incubação, acompanhamento do ciclo de maturidades desses incubados e como a incubadora trabalha no sentido de melhorar a transparência e a padronização dos seus processos. Isso direcionou os respondentes a exporem os processos e práticas utilizados na gestão das incubadoras. Sendo assim, foi possível identificar as categorias para análise, de acordo com os processos-chave da metodologia CERNE NÍVEL 1.

\subsection{Etapa Descritiva}

Analisando o questionário formulado com base nos processos e nas práticas do modelo CERNE NÍVEL 1, pode-se identificar o grau de maturidade que se encontram as incubadoras em cada prática descrita. Como pode ser visto nas figuras a seguir:

Figura 2 - Nível das Práticas-Chave Incubadoras
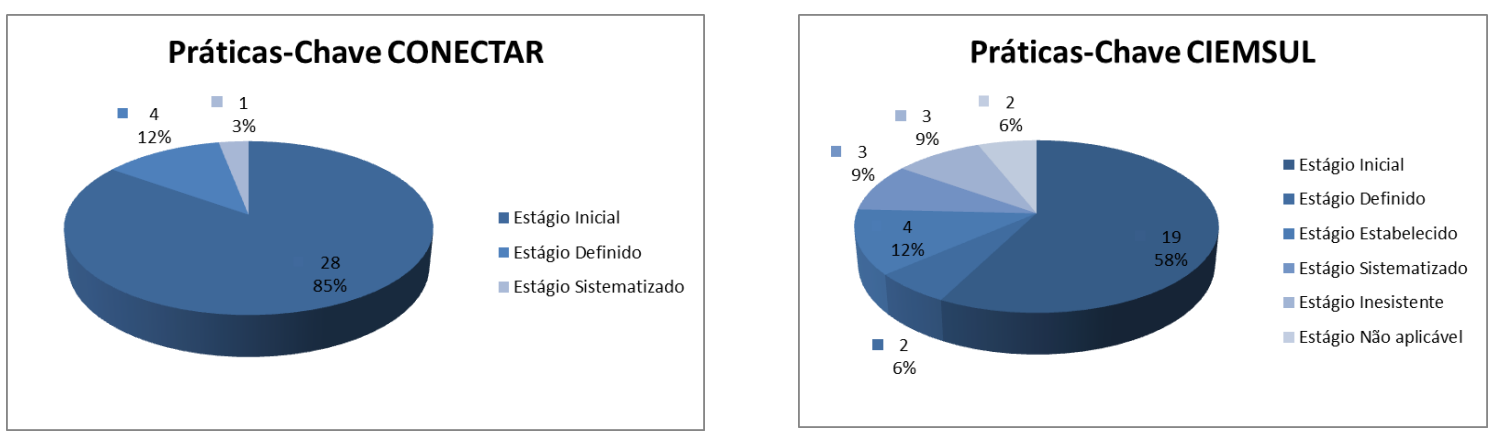

Fonte: Elaborado pelas autoras.

Com base na Figura 2, pode-se identificar as 33 práticas-chave e seus respectivos níveis, componentes do CERNE NÍVEL 1. A incubadora CONECTAR tem $85 \%$ dessas práticas em estágio inicial, já executada, $12 \%$ em estágio definido, possuem planejamento e 3\% em estágio sistematizado, possuem avaliação e aprimoramento. Para a incubadora CIEMSUL, $58 \%$ das práticas estão em estágio inicial, já executadas, $6 \%$ em estágio definido, possuem planejamento, $12 \%$ em estágio estabelecido, com monitoramento, $9 \%$ em estágio sistematizado, possuem avaliação e aprimoramento, $9 \%$ são inexistentes e $6 \%$ não aplicáveis.

Para cada um dos 8 processos-chave do CERNE NÍVEL 1, as incubadoras desenvolvem ações que identificam a implementação das práticas-chave, quanto maior o número de práticas 
implementadas dentro do processo, maior será o nível de maturidade do mesmo. Como pode ser observado na Figura 3:

Figura 3 - Nível dos Processos-Chave Incubadoras
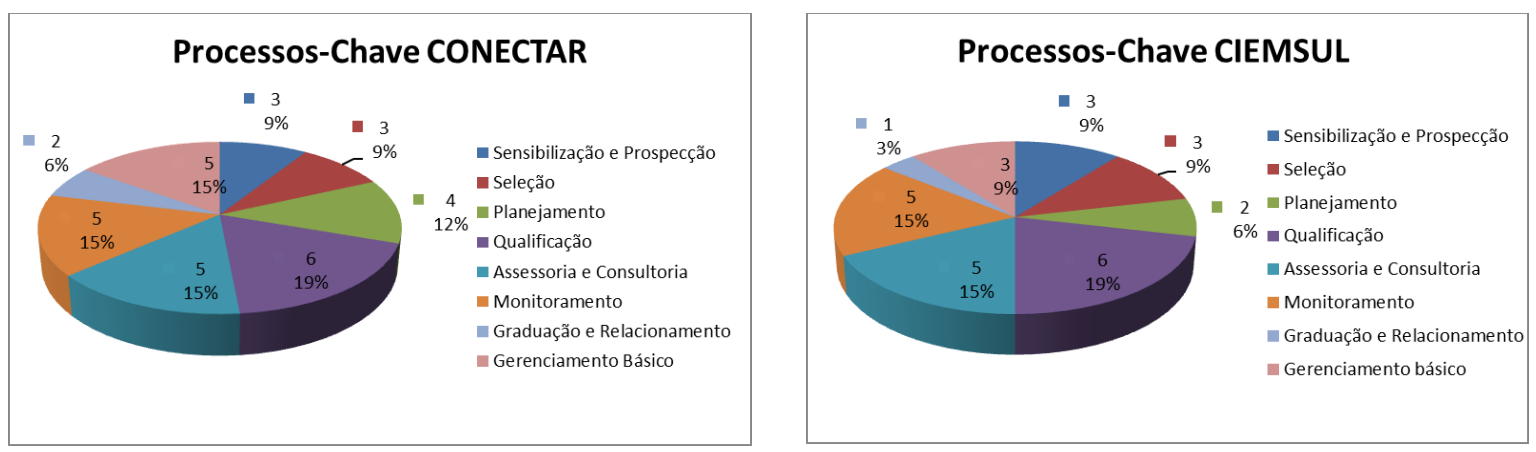

Fonte: Elaborado pelas autoras.

No processo-chave de "Sensibilização e Prospecção", identificou-se que ambas incubadoras possuem 3 práticas implementadas, garantindo assim 9\% do total do CERNE NÍVEL 1. A incubadora CONECTAR promove ações para sensibilização da comunidade, como a "Maratona de Inovação de Pelotas", "Startup Lab Sebrae" e "Startup Weekend". A prospecção ocorre como consequência aos eventos realizados, pelo fato de que os participantes são estimulados a submeter os projetos para a pré-incubação. A qualificação dos empreendedores se dá a partir de workshops e mentorias, em parceria com o Sebrae e com professores e pesquisadores UFPel. Na incubadora CIEMSUL, a divulgação é feita nas salas de aula da universidade, ocasionando assim a sensibilização da comunidade acadêmica e a incubadora também se faz presente em eventos e palestras.

No processo-chave "Seleção", ambas incubadoras possuem 3 práticas implementadas, um percentual de 9\% do CERNE NÍVEL 1 cada. A CONECTAR utiliza edital anual para captação de propostas. Os incubados são submetidos à avaliação sistematizada cujo instrumento utilizado para a referida avaliação ainda não está totalmente definido. Na contratação são recepcionados os integrantes da equipe e é apresentado a eles o contrato com direitos e deveres do incubado. Na CIEMSUL, utiliza-se para processo de seleção de projetos um edital anual. A incubadora possui ferramentas para avaliação dos incubados e a contratação é feita de acordo com o regimento interno de contratos.

Segundo a análise no processo-chave "Planejamento", a incubadora CONECTAR possui 4 práticas implementadas, um percentual de 12\% do CERNE NÍVEL 1. Isso pelo fato de que as necessidades dos incubados são supridas através da demanda dos mesmos em

REMIPE- Revista de Micro e Pequenas Empresas e Empreendedorismo da Fatec Osasco 
determinadas áreas. Também, os empreendedores são orientados a participar de uma mentoria e consultoria específica. Para a CIEMSUL, 2 práticas foram implementadas, totalizando $6 \%$ do CERNE NÍVEL 1. Nesse processo-chave, a incubadora possui práticas inexistentes por não utilizar de uma ferramenta padrão de planejamento.

No processo-chave "Qualificação", ambas incubadoras possuem 6 práticas implementadas, obtendo $19 \%$ do CERNE NÍVEL 1 cada. Na CONECTAR, o incubado é orientado a participar de cursos, palestras e workshps, em parceria com o Sebrae e, esses eventos ocorrem esporadicamente. Na CIEMSUL, os recursos são limitados para ofertar cursos periodicamente para os incubados, sendo possível utilizar os mesmos somente por demanda.

Para o processo-chave de "Assessoria/consultoria", ambas incubadoras possuem 5 práticas implementadas, correspondendo 15\% do CERNE NÍVEL 1 cada. As duas incubadoras não possuem recursos para manter uma consultoria periódica, ocorrendo somente quando há demanda do incubado.

No processo-chave "Monitoramento do Empreendedor", ambas incubadoras possuem 5 práticas implementadas, totalizando $15 \%$ do CERNE NÍVEL 1 . A incubadora CONECTAR faz o monitoramento do incubado em períodos trimestrais em todos os eixos do empreendimento. Para a CIEMSUL, esse é realizado informalmente pelo gestor da incubadora.

No processo-chave de "Graduação e Relacionamento com os Graduados”, a incubadora CONECTAR possui 2 práticas implementadas, um total de $6 \%$ do CERNE NÍVEL 1 . Isso pelo fato de existir regras para a mudança de status definidas no regimento da incubadora. Contudo, essas regras necessitam de alterações para assim promover atividades integradoras entre incubados e graduados. A incubadora CIEMSUL possui 1 prática implementada, totalizando $3 \%$ do CERNE NÍVEL 1. Isso pelo fato de ocorrer à mudança de status de incubado para graduado, mas não há uma prática aplicável para o relacionamento após a graduação.

Segundo análise no processo-chave "Gerenciamento Básico", a incubadora CONECTAR possui 5 práticas implementadas, garantido $15 \%$ do CERNE NÍVEL 1. A incubadora tem sua existência formal comprovada nas instâncias da universidade e possui um gerenciamento financeiro que ocorre por meio do projeto vinculado a fundação Delfim Mendes Silveira. Segundo o coordenador, o modelo de receita necessita de aprimoramento, pois tem captação de recurso baixa. Quanto à estrutura física, hoje a incubadora trabalha em modelo de coworking, alocado no parque tecnológico de Pelotas. Por isso, tem a necessidade de realizar parcerias com laboratórios de pesquisa certificados que colaborem com as empresas incubadas. Outra dificuldade identificada é a sistematização da área de marketing, por falta de um

REMIPE- Revista de Micro e Pequenas Empresas e Empreendedorismo da Fatec Osasco 
profissional específico para a atividade. Na incubadora CIEMSUL 3, práticas foram implementadas, totalizando 9\% do CERNE NÍVEL 1. A incubadora não utiliza uma ferramenta padrão para o planejamento financeiro. A estrutura física é oferecida pelo parque tecnológico, que oferece os serviços de vigilância, limpeza e manutenção. Na área de marketing, a incubadora possui o site para divulgação, mas não possui um profissional específico para a atividade, sendo suprido pelo próprio coordenador.

\subsection{Discussão}

O escopo da metodologia CERNE desenvolvida pelo Sebrae e ANPROTEC demonstra potencial de contribuir para melhorias nos processos de incubação (ANPROTEC, 2014). Segundo os dados apresentados no artigo, identificou-se que as duas incubadoras possuem interesse na implantação da metodologia, sendo observados benefícios a serem conquistados com essa ação, como melhoria na transparência e na padronização dos processos, ampliação da quantidade e da qualidade dos empreendimentos, com o respectivo impacto no aumento da taxa de sucesso.

O CERNE NÍVEL 1 se mostra como um norteador para as incubadoras que estão iniciando o processo de incubação, pois as práticas-chaves para essas incubadoras mostram quais caminhos seguir e quais processos devem adotar. No caso da CONECTAR, onde a maioria das práticas-chave está em estágio inicial, o desenvolvimento das práticas do CERNE NÍVEL 1 o desafio é sistematizar as práticas que já estão sendo adotadas. Já para a incubadora CIEMSUL, que está no mercado há algum tempo, o CERNE NÍVEL 1 se apresenta como um questionador da realidade atual, sendo utilizado para identificar se as práticas realizadas estão sendo feitas da melhor maneira possível. Em ambas incubadoras pode-se observar que a maior parte dos processos é realizada, porém, em alguns casos, não possuem um planejamento prévio, acontecendo de acordo com a demanda dos incubados.

Dessa forma, à luz da implementação do modelo CERNE NÍVEL 1, nenhuma das incubadoras analisadas atenderam plenamente os requisitos do modelo. Entretanto, destaca-se o maior número de respostas positivas para a incubadora CONECTAR. Portanto, há necessidade de um trabalho para a implementação efetiva do modelo em ambas as incubadoras. Isso pode ser feito por meio da articulação de um planejamento de implantação das práticas ainda não adotadas ou que não alcançaram o estágio sistematizado. Esta pesquisa proporcionou, além do conhecimento do ambiente de incubação e sua importância, perceber que o processo

REMIPE- Revista de Micro e Pequenas Empresas e Empreendedorismo da Fatec Osasco V. $6 \mathrm{~N}^{\circ} 2$ jul.-dez. 2020. 
de certificação da qualidade das atividades e serviços prestados pelas incubadoras favorece a busca por boas práticas de gestão. Isso porque reúne processos e práticas como referência para o setor e objetivam o desenvolvimento de empreendimentos inovadores.

\section{CONSIDERAÇÕES FINAIS}

Embora com tempo de atuação diferente, o nível de maturidade das incubadoras analisadas foi similar, na maioria dos processos-chave. No entanto, a diferenciação entre uma e outra se deu no âmbito das práticas desenvolvidas por elas. Das 33 praticas-chaves do CERNE NÍVEL 1 avaliadas no estudo, a incubadora CONECTAR tem 28 práticas no estágio inicial, 4 práticas em estágio definido e 1 prática no estágio sistematizado. A incubadora CIEMSUL, possui 19 praticas-chaves em estágio inicial, 2 práticas em estágio definido, 4 práticas no estágio estabelecido, 3 práticas em estágio sistematizado, 3 práticas em estágio inexistente e 2 práticas não aplicável.

Ao comparar todos os processos-chave e seus respectivos níveis do CERNE, podemos identificar que a incubadora CONECTAR possui 33 práticas implementadas, em sua maioria no estágio inicial, totalizando $100 \%$ do CERNE NÍVEL 1. Na incubadora CIEMSUL, 28 práticas foram implementadas, garantindo um percentual de $85 \%$ do CERNE NÍVEL 1.

Ambas incubadoras possuem limitações para que todas as práticas sejam implementadas e sistematizadas. No caso da CONECTAR, os recursos financeiros são bem limitados, mas por sua vez, possui uma equipe gestora que facilita no seu gerenciamento. Para a CIEMSUL, o fator de maior relevância é sobre a coordenação, todos os processos são desenvolvidos unicamente pelo gestor da incubadora.

Uma questão limitadora desta pesquisa foi o fato de não ter sido possível abranger todas as incubadoras do Extremo Sul do Rio Grande do Sul, tendo ficado de fora a incubadora INNOVATIO. Sugere-se como estudos futuros, a realização da pesquisa em um número maior de incubadoras, de outras regiões, o que possibilitará a comparação dos dados. Adicionalmente, não se buscou esgotar todos os assuntos relacionados às incubadoras de empresas e o processo de certificação, pelo fato de que o modelo CERNE está sendo reestruturado, o que gera possíveis apontamentos para pesquisas futuras. 


\section{REFERÊNCIAS}

ANPROTEC. Sumário Executivo. Centro de referência para apoio a novos empreendimentosCERNE, Brasília, DF, Brasil, 2015. Disponível em: <http://www.anprotec.org.br/Relata/20141211_Anprotec_Certificacao_SumarioExecutivo_Ba ixa.pdf $>$. Acesso em: 11 nov 2018.

. Disponível em <http://www.anprotec.org.br> Acesso em: 19 out 2018. em: 10 set.2018.

Disponível em:<http://anprotec.org.br/site/publicacoes-anprotec/ebooks/> Acesso

Estudo de Projetos de Alta Complexidade: indicadores de parques tecnológicos / Centro de Apoio ao Desenvolvimento Tecnológico. Ministério da Ciência, Tecnologia e Inovação $\quad-\quad$ Brasília: $\quad$ CDT/UnB, $2014 . \quad$ Disponível em: $<$ http://www.anprotec.org.br/Relata/PNI_FINAL_web.pdf $>$ Acesso em: 15 set 18.

Panorama 2001. Brasília:2001. 42p.

ANPROTEC; SEBRAE; CERNE - Centro de Referência para Apoio à Novos Empreendimentos: Termo de Referência. Brasília, 2011.

ANPROTEC; MCTI. Estudo, Análise e Proposições sobre as Incubadoras de Empresas no Brasil. Brasília, ANPROTEC, 2011.

Estudo, Análise e Proposições sobre as Incubadoras de Empresas no Brasil. Brasília, ANPROTEC, 2012.

AUDY, Jorge Luis Nicolas. Dos parques científicos e tecnológicos aos ecossistemas de inovação: Desenvolvimento social e econômico na sociedade do conhecimento. Brasília, DF :

BELLAVISTA, Joan; SANZ, Luis. Science and technology parks: habitats of innovation: introduction to special section. Science and Public Policy, 36(7), p. 499-510, 2009. Disponível em: $\quad<\quad$ https://academic.oup.com/spp/articleabstract/36/7/499/1619902?redirectedFrom=fulltext $>$.

BLANK, Steve; DORF Bob. Startup: Manual do empreendedor. O guia do passo a passo para construir uma grande empresa. São Paulo: Alta Books, 2014.BARDIN, Laurence. Análise de conteúdo. São Paulo: 2011.Edições 70.

CIEMSUL. Disponível em: < http://ciemsul.ucpel.edu.br/institucional >. Acesso em: 30 out 2018

CONECTAR. Disponível em: <https://wp.ufpel.edu.br/conectar/historicoquem-somos/>. Acesso em: 30 out 2018.

CHIAVENATO, I. Empreendedorismo: dando asas ao espírito empreendedor. 2ed. São Paulo: Saraiva, 2007.

DOLABELA, Fernando. O segredo de Luísa: uma idéia, uma paixão e um plano de negócios: como nasce o empreendedor e se cria uma empresa. 14. ed. São Paulo: Cultura, 2006. 312p.

REMIPE- Revista de Micro e Pequenas Empresas e Empreendedorismo da Fatec Osasco V. $6 \mathrm{~N}^{\circ} 2$ jul.-dez. 2020. 
DORNELAS, José Carlos Assis. Empreendedorismo corporativo: como ser empreendedor, inovar e se diferenciar na sua empresa. 2. ed. Rio de Janeiro: Elsevier, 2008. xii, 166 p.

HASSINK, Robert; HU, Xiaohui. Specialisation to Diversification in Science and Technology Parks. World Technopolis Association, WTR, 1, p. 6-15, 2012. Disponível em:< https://www.researchgate.net/publication/271137161_From_Specialisation_to_Diversification _in_Science_and_Technology_Parks> .

LIMEIRA, Tania Vidigal. O papel das aceleradoras de impacto no desenvolvimento dos negócios sociais no Brasil. São Paulo: FGV, 2014.

MINISTÉRIO DA CIÊNCIA, TECNOLOGIA E INOVAÇÃO - MCTI. Programa Nacional de Apoio às Incubadoras de Empresas e aos Parques Tecnológicos - PNI. Portaria $\mathbf{n}^{\circ} 139$. Brasília, 2009.

MORAIS, E.F.C. Seminário de Inovação 2007. Disponível em:

<http://www.mbc.org.br/mbc/uploads/bibliotec/1182775933.6469a.PDF> Acesso em : 22 set 2018.

NBIA - National Business Incubation Association. State of the Business Incubation Industry. Athens, Ohio: NBIA Publications, 2012. Disponível em: $\langle$ https://www.nbia.org/resource_library >

OLIVEIRA, José Rodrigues. Incubadoras Universitárias de empresas e de cooperativas: Contrastes e desafios. São Paulo: UNICAMP, 2003. Dissertação (Mestrado em Política Científica e Tecnológica ) -Universidade Estadual de Campinas, 2003.

PLONSKI, Guilherme Ary.Bases para um movimento pela inovação tecnológica no Brasil. Revista São Paulo em Perspectiva. V.19, p.25-33,2006. Disponìvel em : $<$ http://produtos.seade.gov.br/produtos/spp/v19n01/v19n01_02.pdf>. Acesso em : 22 set.2018.

RIES, Eric. A Startup Enxuta: Como os empreendedores atuais utilizam a inovação contínua para criar empresas extremamente bem-sucedidas. São Paulo: Leya, 2012.

SEBRAE - SERVIÇO BRASILEIRO DE APOIO ÀS MICRO E PEQUENAS EMPRESAS. Sobrevivência das Empresas no Brasil. Brasília: Sebrae, out 2016. Disponível em: $<$ http://www.sebrae.com.br/Sebrae/Portal\%20Sebrae/Anexos/sobrevivencia-das-empresas-nobrasil-102016.pdf > Acesso em: 27 set 2018.

TIDD, Joe Tidd; BESSANT, Joe Bessant. Gestão da inovação. 5. ed. Porto Alegre: Bookman,2015.p.8-10.Disponível

em: $<$ https://books.google.com.br/books?id=Jyj1BgAAQBAJ\&printsec=frontcover\&hl=pt-

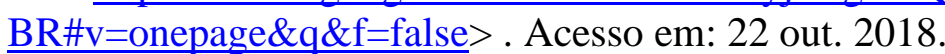

REMIPE- Revista de Micro e Pequenas Empresas e Empreendedorismo da Fatec Osasco V. $6 \mathrm{~N}^{\circ} 2$ jul.-dez. 2020. 